\title{
Habilidades numéricas básicas: Algumas contribuições da análise do comportamento
}

\author{
Angela Bernardo de Lorena \\ Universidade Federal de São Carlos \\ Instituto Nacional de Ciência e Tecnologia sobre Comportamento, Cognição e Ensino \\ Janaina de Fátima Castro-Caneguim \\ Universidade Federal de São Carlos \\ João dos Santos Carmo \\ Universidade Federal de São Carlos \\ Instituto Nacional de Ciência e Tecnologia sobre Comportamento, Cognição e Ensino
}

\begin{abstract}
Resumo
Diversos estudos investigaram as habilidades numéricas básicas na espécie humana, tanto as de origem filogenética quanto as aprendidas a partir das experiências culturais. O papel dessas habilidades na aquisição e desenvolvimento de repertórios matemáticos complexos foi destacado nessas pesquisas. O objetivo deste artigo foi oferecer alguns dados fundamentais sobre aquisição de habilidades numéricas básicas, discutindoos a partir do referencial da Análise do Comportamento. O estudo é uma revisão ampla da literatura e parte da descrição de dados externos às pesquisas comportamentais, abrangendo senso numérico, subitização e contagem, buscando-se chegar à proposta de comportamento conceitual numérico. Também foram selecionados e descritos os principais trabalhos sobre essas habilidades a partir da Análise do Comportamento. Ao final, implicações para pesquisas e aplicações em ambientes educacionais são oferecidas.
\end{abstract}

Palavras-chave: habilidades numéricas básicas; senso numérico; subitização; conceito de número; análise do comportamento.

\begin{abstract}
Basic numerical skills: Some contributions from behavior analysis. Several studies have investigated the basic numerical skills in the human species, both from a phylogenetic origin and from cultural experiences. These investigations highlighted the role of numerical abilities in the acquisition and development of complex mathematical repertoires. The aim of this article was to offer some fundamental data about the acquisition of basic numerical skills, which are discussed from a behavioral point of view. The study is a broad review of the literature and starts on the description of external data to behavioral research, including number sense, subitizing and counting in order to present the proposal of numeric conceptual behavior. Some major behavioral works on these skills were also selected and described. Finally, some implications to research as well as to educational settings are offered.
\end{abstract}

Keywords: basic numerical skills; number sense; subitizing; number concept; behavior analysis.

\section{Resumen}

Habilidades numéricas básicas: Algunos aportes del análisis de la conducta. Varios estudios han investigado las habilidades numéricas básicas en la especie humana, tanto el origen filogenético como las extraídas de experiencias culturales. El papel de estas competencias en la adquisición y desarrollo de repertorios matemáticas complejas se puso de relieve en estos estudios. El propósito de este artículo es proporcionar algunos datos básicos sobre la adquisición de las habilidades numéricas básicas, y discutirlas desde en punto de vista de la Análisis de la Conducta. El estudio es una amplia revisión de la literatura y de la descripción de datos externos a la investigación conductual, incluyendo el sentido numérico, subitización y conteo, tratando de alcanzar la propuesta de comportamiento conceptual numérico. También se han seleccionado y descrito los principales trabajos en estas habilidades desde el Análisis de la Conducta. Al final, se ofrecen implicaciones para la investigación y las aplicaciones en entornos educativos.

Palabras clave: habilidades numéricas básicas; sentido numérico; subitización; concepto de número; análisis de la conducta. 
$\mathrm{A}$ matemática vem sendo praticada e ensinada em diferentes contextos e configura-se como uma disciplina importante na vida contemporânea, assim como foi importante para o desenvolvimento da sociedade humana. Apesar dessa importância constatada e da íntima relação que essa área do conhecimento tem com atividades que são praticadas cotidianamente (como, fazer compras, calcular o tempo que se deve deixar uma massa de pão descansando para depois colocála para assar), há inúmeros casos de fracasso na aprendizagem formal da matemática. A partir de um amplo estudo de revisão sobre aquisição de habilidades numéricas em crianças, Sophian (1996) aponta para uma diferença marcante entre a matemática ensinada na escola e a matemática praticada pelas crianças fora da escola. No segundo caso, de acordo com Sophian, as crianças costumam se divertir com brincadeiras que envolvem números. Canções, jogos, parlendas, atividades livres como contar as estrelas etc., tornam prazeroso o contato com o mundo dos números. Em relação à escola, porém, as crianças começam a aprender que a matemática não é algo tão prazeroso e que elas não são tão boas nesse assunto quanto julgavam ser. Sophian, então, levanta os seguintes questionamentos: (a) porque algumas coisas que fazemos com números são tão divertidas e outras tão aversivas? (b) as atividades matemáticas que as crianças encontram em sala de aula são fundamentalmente diferentes das que encontram lá fora? É possível tornar a matemática escolar mais divertida e mais compreensível?

Infelizmente a disparidade entre a matemática escolar e os divertidos jogos matemáticos da vida diária infantil, apontados por Sophian (1996), denunciam uma questão extremamente grave: nossas crianças são expostas a experiências escolares que estão longe de tornar a matemática algo prazeroso e, muito menos, um instrumento para investigação, descoberta $\mathrm{e}$ conhecimento sobre os fatos do mundo e da natureza. Nunes e Bryant (1996) sugerem que em uma sociedade complexa como a nossa, as crianças precisam ser "numeralizadas", significando com isso que elas sejam capazes de pensar e discutir acerca de relações numéricas e espaciais usando as ferramentas de sua cultura. Em resumo, Nunes e Bryant propõem que as crianças precisam ser ensinadas a usarem as regras lógicas, os sistemas numéricos convencionais e a aplicarem o raciocínio matemático significativamente e apropriadamente em diferentes situações cotidianas. Essas conclusões de Nunes e Bryant corroboram afirmações prévias acerca da aprendizagem de habilidades numéricas em crianças pequenas, desde o conceito de número até operações básicas (Kamii \& Housman, 2002).

Embora a apropriação e a aplicação do conhecimento matemático não devam ser encaradas como uma tarefa exclusiva da escola, os professores necessitam de um preparo e formação que ofereçam suporte diferenciado ao seu trabalho. Além disso, necessitam de informações pautadas em resultados de pesquisas acerca de como as crianças adquirem não apenas os conteúdos trabalhados nas escolas, mas, sobretudo, como desenvolvem as habilidades numéricas iniciais, cujas bases podem ser encontradas bem antes das primeiras incursões na vida escolar. É objetivo do presente artigo, apresentar uma breve revisão dos principais trabalhos acerca das habilidades numéricas, estas entendidas como comportamentos desenvolvidos ao longo da história filogenética de nossa espécie, porém modelados a partir das contingências ambientais presentes em uma dada cultura. Será oferecida uma leitura comportamental de alguns estudos sobre senso numérico, subitização e contagem, bem como será exposta uma proposta de tratamento do conceito de número enquanto comportamento conceitual numérico. O texto partirá de dados externos às pesquisas comportamentais a fim de se chegar à proposta de comportamento conceitual numérico e algumas de suas implicações ao campo educacional.

No presente texto, portanto, consideraremos como habilidades numéricas básicas (ou elementares) aquelas capacidades que são cruciais para o aprendizado da matemática: a subitização, o senso numérico, a contagem e as relações contidas no comportamento conceitual numérico. Serão descritos e discutidos a seguir os três primeiros conceitos separadamente.

\section{As habilidades numéricas básicas}

Os comportamentos ou habilidades numéricas são aqueles repertórios que ocorrem em contextos que envolvem numerosidade, problemas aritméticos, estimativas, cálculos, etc. Em qualquer atividade humana, apresentar essas habilidades parece ser crucial para a execução de algumas tarefas no diaa-dia.

Subitização. Subitização (neologismo em inglês, subitizing, cuja raiz encontra-se na palavra "súbito") é a capacidade de discriminar subitamente pequenas numerosidades (até três ou quatro elementos) e de responder discriminativamente a pequenas alterações (acréscimos ou retiradas) no número total de elementos de uma coleção, de forma muito rápida milésimos de segundos. A capacidade de subitizar parece ser parte de nossa espécie. Bebês humanos de poucas semanas, sob condições controladas, são capazes de reagir a mudanças na quantidade dos elementos de uma coleção, mesmo sendo pré-verbais, e, portanto, sem terem aprendido a contar, somar ou subtrair (Wynn, 1998). A subitização, nesse caso, parecer estar relacionada a aquisições filogenéticas e é apropriadamente chamada de subitização perceptiva (Clements, 1999). Por outro lado, crianças verbais e adultos humanos também conseguem subitizar pequenas quantidades de elementos de uma coleção, discriminando acertadamente uma dada quantidade e nomeando-a adequadamente. Nesse caso, a subitização, embora de origem filogenética, está diretamente relacionada à experiência social de contar e nomear a quantidade de elementos, e é chamada de subitização conceitual (Clements, 1999). Em nossa espécie, discriminar adequadamente quantidades acima de cinco ou seis elementos é uma tarefa difícil, principalmente quando outras variáveis estão envolvidas (distribuição aleatória dos elementos em contraposição à distribuição canônica; densidade dos elementos no espaço; sobreposição dos elementos; tipo de elementos de uma coleção). Nos casos em que há coleções com grandes quantidades, crianças e adultos verbais utilizam a estimativa, que é uma capacidade derivada da subitização, geralmente estabelecendo julgamentos bastante próximos da quantidade exata de elementos.

Hauser e Spelke (2004), ao revisar os estudos de subitização 
em bebês humanos e outros primatas, identificaram os aspectos cruciais que possibilitam a subitização: 1) a quantidade a ser identificada de conter no máximo quatro elementos; 2) os elementos devem estar em posições distintas, ou seja, sem sobreposição; 3) os elementos estar separados, isto é não conectados; 4) devem estar estático ou em movimento contínuo. Se algumas destas condições não estiverem presentes, é possível que a subitização não ocorra, isto é, não será possível identificar a quantidade de elementos de forma tão rápida.

Subitizar é apontada como uma capacidade filogeneticamente determinada que responderia pelos resultados encontrados nos estudos de discriminação de quantidades em bebês humanos; alguns autores defendem que ela estaria na origem de capacidades mais complexas como soma e subtração. Portanto, a compreensão da capacidade de subitizar parece ser essencial para esclarecer tanto as habilidades numéricas elementares como habilidades numéricas mais complexas.

Senso numérico. A expressão senso numérico (também chamada de sentido de número ou ainda sentido numérico), tal como subitização, é uma tradução do inglês "number sense". Segundo Corso e Dorneles (2010), não há consenso entre os autores sobre a definição exata do que seria o 'senso numérico'. Para estas autoras, essa capacidade "se refere à facilidade e à flexibilidade das crianças com números e à sua compreensão do significado dos números e idéias relacionadas a eles" (p. 229). Essa definição, entretanto, parece muito ampla. Lipton e Spelke (2003) preferem restringir o uso da expressão à capacidade de responder a magnitudes numéricas aproximadas e argumentam que adultos humanos verbais, que sabem contar, quando diante de coleções com muitos elementos comumente se vêem limitados na contagem exata e lançam mão de estimativas aproximadas. Essa habilidade, segundo Lipton e Spelke, está baseada numa sensibilidade à numerosidade, que é o senso numérico. Como se vê, senso numérico e subitização são processos muito próximos e, de certa forma, apresentam características imbricadas entre si.

Também não há acordo, ainda, entre os pesquisadores sobre a origem dessa capacidade. Alguns deles sugerem que existiria uma predisposição inata para apresentarmos tal capacidade (Dehaene, 1997; Hauser \& Spelke, 2004). Pesquisas foram realizadas para investigar essa hipótese, como os estudos conduzidos por Karen Wynn, em bebês humanos, demonstrando que já nascemos com a capacidade de diferenciação de quantidades pequenas e de operações de acréscimo e retirada de objetos (um tipo rudimentar de soma e subtração). Wynn (1992) divulgou dados de discriminação de quantidades pequenas presentes em bebês de cinco meses e num estudo posterior (McCrink \& Wynn, 2004), as habilidades de percepção de acréscimo e retirada de elementos foram identificadas em bebês a partir de oito meses de vida.

Já outros autores, que defendem uma perspectiva desenvolvimentista do senso numérico, acreditam que a "formação de categorias conceituais de número" ocorreria a partir do nascimento. Em outras palavras, essas habilidades observadas em bebês nada mais são do que processos gerais da percepção, aliados com as interações da própria cultura (Barbosa, 2007; Fuson, 1988; Mix \& Sandhofer, 2007).
O senso numérico, enquanto capacidade de compreensão de situações que envolvem números (e.g., quantificar, medir, relacionar, comparar, estimar), além de parecer muito importante para a sobrevivência da espécie, também tem sido entendido como um pré-requisito para habilidades mais complexas a serem aprendidas na escola, como resolução de cálculos e equações (Souza, 2008). No entanto, pouco tem se discutido na literatura sobre a representação multissensorial do senso numérico, como foi relatado por Jordan e Brannon (2006), que argumentam ser possível que todos os sentidos (visão, olfato, tato, paladar e audição) também compartilhem deste mesmo princípio. No artigo destes autores, eles discutem a possibilidade do desenvolvimento e evolução de um sistema antigo para uma representação não-verbal de número, quando demonstraram que podem ser feitas relações numéricas entre estímulos vistos e estímulos ouvidos.

O estudo de Jordan e Brannon (2006) traz uma reflexão importante, pois a maioria dos estudos sobre senso numérico tem focado capacidade de discriminação visual de elementos de um conjunto. Mas, se é de fato há um sistema rudimentar da representação numérica, poderíamos imaginar que se trata de uma capacidade compartilhada entre as diferentes modalidades perceptivas.

Contagem. Como sabemos que uma criança já sabe o que é número? É bastante comum que, desde pequenas, crianças sejam capazes de mostrar corretamente o número de dedinhos quando lhe é perguntado: “quantos anos você tem?"; também não é incomum observarmos crianças recitando "um-dois-três...". Mas o quanto isso quer dizer que a criança entende o que é número?

A aquisição do comportamento conceitual numérico, conforme será visto mais adiante, já está bem documentada nos estudos em análise do comportamento, porém pouco divulgada. Porém, a habilidade de contar e seu papel na aquisição do comportamento conceitual numérico ainda é um tema controverso. Há dados que indicam a contagem como tendo um papel decisivo para esta aquisição, outros dados contradizem essa suposição.

A contagem é, em si, uma habilidade complexa composta por diferentes aquisições que, em conjunto, possibilitam a identificação da quantidade de elementos em uma coleção. Os analistas do comportamento se beneficiaram das proposições de Gelman e Gallistel (1978) unicamente no que diz respeito à descrição operacional do comportamento de contar, rejeitando os princípios inatistas da proposta, uma vez que estes autores defenderiam que a contagem dependeria de habilidades inatas, posição não compartilhada pela Análise do Comportamento.

Desse modo, baseado na descrição de Gelman e Gallistel (1978), a contagem envolve as seguintes sub-habilidades: (a) produção de cadeia verbal numérica (palavras-número): a recitação da sequência dos nomes dos números em uma dada ordem estável (um, dois, três, quatro...) é uma primeira aquisição, fundamental à aprendizagem das demais sub-habilidades; (b) estabelecimento de relação termo-a-termo: a correspondência um para um (ou seja, cada nome de número da sequência numérica verbal corresponde um e somente um elemento da coleção a ser contada), é outra aquisição fundamental. Para 
ser emitida essa correspondência termo-a-termo é necessário que a produção da cadeia verbal siga uma ordem estável, sem repetição dos nomes dos números e sem repetição do elemento relacionado à palavra-número. Produzir a sequência numérica verbal, relacionando cada elemento a um e somente um objeto, no entanto, não esgotam a habilidade de contar; (c) a cardinação é a aquisição fundamental: isto é, a noção de que o último elemento contado indica a quantidade total de elementos da coleção, desde que respeitadas a produção da sequência verbal numérica em uma ordem estável e a correspondência termoa-termo. Evidentemente que a noção de cardinação é mais complexa e envolve a inclusão de classes numéricas. Assim, o número três, por exemplo, representa uma classe numérica que envolve a classe do "dois" e a classe do "um". Porém, essa noção de inclusão de classe será adquirida com o tempo, até que a criança aprenda a relacionar o último elemento contado à quantidade final de elementos da coleção. Essas três subhabilidades são indicativos suficientes para afirmarmos que uma criança já adquiriu a noção de contagem, mas nossa cultura letrada modela três outras sub-habilidades complementares; (d) irrelevância da ordem: pode-se contar a partir de qualquer elemento da coleção e, a cada contagem, pode-se seguir outra ordem qualquer, desde que as três primeiras sub-habilidades sejam respeitadas; (e) generalização e abstração: pode-se contar qualquer coisa, uma vez que o ato de contar deve estar sob controle da numerosidade, que é uma propriedade abstrata da relação entre elementos; (f) finalmente, podem-se agrupar os elementos contados e convencionar-se uma representação para indicar um determinado agrupamento. Assim, por exemplo, se convencionarmos que levantaremos um dedo para cada cinco coisas contadas e, ao final da contagem, estivermos com quatro dedos levantados, poderemos indicar com segurança que foram contados vinte coisas.

Prado (2010), ao descrever diversas pesquisas que buscam identificar o papel da contagem, aponta que alguns resultados indicaram que fatores podem facilitar ou dificultar a contagem de crianças por volta dos quatro anos, como por exemplo: a distribuição espacial dos objetos pode dificultar a contagem, se houver mais de cinco elementos, bem como se os objetos forem móveis.

De qualquer forma, o que precisa ficar claro é que quando nos referimos a número não estamos falando do numeral, que é a representação gráfica do número. Número é uma idéia abstrata que envolve um conjunto de relações entre numeral, quantidade, palavra escrita, etc. ${ }^{1}$

Essa capacidade de contar elementos, a partir dos critérios estabelecidos, só foi demonstrada até agora por humanos, segundo Hauser e Spelke (2004), que descreveram pesquisas nas quais eles buscam identificar se esta capacidade estaria presente em primatas não-humanos que guardam uma grande proximidade genética com nossa espécie.

A aprendizagem cultural e particularmente a aprendizagem do comportamento verbal exercem grande interferência na aquisição das habilidades matemáticas mais complexas e, também, na contagem (Staats \& Staats, 1973). Partindo da concepção proposta por Skinner de que a matemática deveria ser entendida como comportamento verbal, Staats e Staats elaboraram um breve ensaio que denominaram de desenvolvimento de sequências de linguagem matemática. Para estes autores, a matemática deveria ser analisada da mesma forma que outros comportamentos verbais, os quais envolvem tateio de objetos e de outros estímulos verbais, aquisição de sequências verbais e outros repertórios, seguindo os mesmos princípios já suficientemente investigados em laboratório. Os autores contribuíram para a área afirmando que o comportamento de contar era composto por "tato, sequências de resposta-número e extensões através de redundância". Respostas-número são a verbalização do nome de um número na presença da quantidade correspondente de objetos ou do algarismo correspondente, estabelecidas através do treino de tato. Tatear números pode ser aprendido através do ensino de discriminações que resulta em abstração, isto é, "uma resposta sob controle de uma única propriedade isolada de um estímulo, que não pode existir isoladamente" (p. 242). Após (ou até simultaneamente) o estabelecimento dos primeiros tateios em relação aos numerais, a comunidade verbal ensina o encadeamento verbal ou sequencia verbal dos números. Para Staats e Staats (1973), a contagem inicia-se, provavelmente, pela aquisição da sequencia verbal dos números. Posteriormente, a comunidade verbal incentivaria respostas manuais e de seguimento com os olhos, da esquerda para a direita, caso os objetos ou símbolos estejam alinhados. Situações adicionais de ensino diante de grupos de objetos em arranjos diversificados também seriam necessárias para a aprendizagem da contagem.

Conforme salientaram Ribeiro, Assis e Enumo (2007), a aprendizagem da matemática está relacionada à aquisição da linguagem e outros comportamentos complexos que ainda precisam ser mais bem esclarecidos, em relação aos princípios básicos do comportamento. Serão necessárias muitas pesquisas para esclarecer o que são esses processos e como eles evoluem para a aquisição das habilidades numéricas básicas.

Embora ainda sejam necessárias mais pesquisas para elucidar os pormenores destes processos envolvendo a evolução das habilidades numéricas em humanos, já podemos afirmar com alguma segurança que temos uma sensibilidade quantitativa, o que nos permite, desde muito cedo, distinguir quando há mais ou menos objetos, de maneira praticamente automática. Isso significa que somos capazes de perceber pequenas numerosidades sem que isso dependa de algum processo de aprendizagem formal.

A aquisição do senso numérico e da contagem na educação infantil são fortes preditores do desempenho em matemática nos primeiros anos escolares (Corso \& Dorneles, 2010). Essas noções são fundamentais para o planejamento de ensino e para a intervenção junto a indivíduos com dificuldades acentuadas de aprendizagem. Conforme afirmamos anteriormente, os estudos sobre subitização, senso numérico e contagem fornecem contribuições significativas de outras abordagens teóricas, e os analistas do comportamento aos poucos vão se apropriando de alguns dados da literatura. Em acréscimo a essas contribuições externas, os estudos em Análise do Comportamento têm produzido dados relevantes sobre o que tem sido tradicionalmente chamado de conceito de número. Essa expressão tem sido substituída por comportamento conceitual numérico e alguns dados de pesquisa 
e suas implicações educacionais serão apresentados a seguir.

\section{Comportamento conceitual numérico: algumas contribuições da análise experimental do comportamento}

Carmo (2002) propõe que o termo conceito de número seja substituído por comportamento conceitual numérico, tendo em vista que: (a) conceito de número remete a processos cognitivos e, portanto, internos ao indivíduo, o que dificulta a identificação de relações mais amplas da interação indivíduo-ambiente, como sugere a análise do comportamento; (b) enquanto comportamento conceitual numérico enfatiza as relações mantidas entre o indivíduo e os eventos ambientais, possibilitando descrever operacionalmente as respostas que comporiam uma rede de relações numéricas equivalentes. Além disso, propõe que não há somente um comportamento conceitual numérico, e sim comportamentos conceituais numéricos cujos componentes são gerados e mantidos pela cultura.

A rigor, a proposição de Carmo (2002) quanto ao uso do termo comportamento conceitual numérico tem base em uma série de estudos que, em diferentes momentos, confirmam a noção de que precisamos, cada vez mais, identificar as variáveis cruciais das quais o comportamento numérico é função.

Em se tratando de um tipo particular de comportamento matemático, que chamaremos de comportamento conceitual numérico, podemos dividir as contribuições da análise do comportamento em: (a) estudos experimentais básicos com crianças pré-escolares que apresentam desenvolvimento típico; (b) estudos experimentais básicos com indivíduos que apresentam desenvolvimento atípico. Outra forma de classificar esses estudos é: (a) com a aplicação do paradigma de equivalência de estímulos (conforme proposição de Sidman \& Tailby, 1982); (b) sem a aplicação do paradigma de equivalência de estímulos. Esses estudos, independentemente do paradigma utilizado, possuem como característica comum o uso de procedimentos experimentais derivados de uma tecnologia de controle de estímulos ${ }^{2}$.

Antes de apreciarmos essas contribuições, é importante destacar que o paradigma de equivalência proposto por Sidman e Tailby (1982) trouxe implicações fundamentais ao entendimento da aquisição de conceitos e do comportamento simbólico (Mcllvane, Galvão, Goulart, Brino, \& Barros, 2005). Resumidamente, equivalência de estímulos é um modelo teórico que permite prever se um estímulo será incluído em uma classe de estímulos que se equivalem, a qual se caracteriza pela intercâmbio de funções entre os estímulos a ela pertencentes e é formada a partir de relações condicionais arbitrariamente estabelecidas entre o estímulo a ser incluído e um ou mais membros dessa classe.

Tal modelo é definido com base na presença de três propriedades extraídas da teoria dos conjuntos: reflexividade, simetria e transitividade. Na reflexividade um determinado elemento relaciona-se consigo mesmo, ou seja, $a$ relaciona-se a $a$. Em notação matemática, diríamos $a \mathrm{R} a$, onde $\mathrm{R}$ significa relação. Para que uma relação seja simétrica é preciso que dois elementos, relacionados numa certa ordem, estejam relacionados na ordem inversa, isto é, se $a \mathrm{R} b$, então $b \mathrm{R} a$. Na transitividade, estando dois elementos relacionados a um elemento em comum, esses dois elementos devem estar relacionados entre si, ou seja, se $a \mathrm{R} b$ e $b \mathrm{R} c$, então $a \mathrm{R} c$. Notemos que o mesmo é válido para uma relação transitiva cujos membros estão relacionados na seguinte ordem: se $a \mathrm{R} b$ e $a \mathrm{R} c$, então $b \mathrm{R} c$. Ou seja, a transitividade no paradigma de equivalência não segue necessariamente a mesma ordem transitiva da matemática. Além disso, nos estudos experimentais tem sido conduzido um teste final de equivalência no qual é verificada a emergência da relação simétrica da transitiva, ou seja, $c \mathrm{R} a$ ou, no segundo caso, $c \mathrm{R} b$.

Dessa forma, esboça-se uma possibilidade de análise funcional do que temos aqui denominado de comportamento conceitual em termos de rede complexa de relações entre eventos ambientais e eventos comportamentais, e entre eventos ambientais que passam a fazer parte de uma mesma classe de estímulos equivalentes ${ }^{3}$. Subjacente a esta rede de relações, estão os processos de discriminação, generalização, abstração e equivalência de estímulos.

Os estudos experimentais com base no paradigma de equivalência tipicamente lançam mão de um procedimento simples e altamente eficaz na investigação de comportamentos relacionais: emparelhamento ao modelo ou matching to sample (MTS). Esse procedimento consiste em apresentar um estímulo modelo e, a seguir, alguns estímulos de comparação, também chamados de estímulos de escolha (usualmente o número de comparações varia de dois a três estímulos e podese, opcionalmente, requisitar uma "resposta de observação" ao modelo, antes de apresentar os estímulos de escolha. Esta resposta pode ser tocar, apontar, dizer o nome do estímulo, ou qualquer outra que indique que o sujeito observou o modelo). A tarefa do sujeito é escolher, dentre as comparações, qual se relaciona ao modelo, por algum critério arbitrário (semelhança física, dimensional, funcional, etc.).

Os estudos experimentais básicos com crianças que apresentam desenvolvimento típico acrescentaram dados fundamentais ao entendimento da aquisição do comportamento conceitual numérico. Um estudo pioneiro foi conduzido por Drachenberg em 1973 e reeditado em 2010. O objetivo foi ensinar o conceito de número para crianças na fase pré-escolar por meio do procedimento de MTS. Segundo Drachenberg, muitos problemas escolares, relativos ao processo de ensinoaprendizagem da aritmética durante a escola primária, teriam conexão com a formação incompleta ou ausência na formação de conceitos básicos nessa área. Portanto, uma adequada aprendizagem do conceito de número por pré-escolares seria importante para garantir a aprendizagem efetiva da aritmética no decorrer das séries iniciais do Ensino Fundamental. O trabalho de Drachenberg destaca a efetividade do MTS no estabelecimento de comportamentos relacionais envolvendo numerais e quantidades.

No que diz respeito ao ensino do conceito de quantidade, crucial para a aquisição de comportamento conceitual numérico, Kahhale (1993) objetivou verificar a efetividade do procedimento de discriminação sem erro, além de investigar como o conceito de quantidade poderia ser ampliado com os procedimentos de equivalência de estímulos. Kahhale (1993) usou o procedimento de MTS para ensinar a correspondência termo-a-termo entre dois 
conjuntos de estímulos a crianças com idade entre cinco e sete anos, e manipulou as variáveis irrelevantes dos elementos dos conjuntos (tamanho, cor, forma, densidade) de maneira a isolar a variável crucial que é a numerosidade.

Outros estudos mostraram que o MTS e, mais especificamente, o paradigma de equivalência oferecem vantagens substanciais ao estudo da aquisição de comportamento conceitual numérico (Carmo \& Galvão, 1999; De Leon, 1998). Outras investigações baseadas na tecnologia de controle de estímulos, porém sem o uso do paradigma de equivalência, mostraram-se fundamentais para: (a) construção de currículos funcionais para pré-escolares e alunos das séries iniciais (Beckwith et al., 1979; Resnick, Wang, \& Kaplan, 1973; Teixeira, 2010); (b) identificação, descrição e entendimento da aquisição de habilidades isoladas que fazem parte da rede de relações componentes do comportamento conceitual numérico; por exemplo, ordenação (Assis \& Costa, 2004); discriminação de quantidades (Almeida, Arantes, \& Machado, 2007); contagem (Diaz \& García, 1980; González \& García, 1984; Magalhães \& Galvão, 2010; Monteiro \& Medeiros, 2002; Schoenfeld, Cole, \& Sussman, 1976).

Em relação aos estudos envolvendo indivíduos com alguma limitação cognitiva ou sensorial, destaca-se a investigação conduzida por Green em 1993 e reeditada em 2010. Green estudou a equivalência entre números e quantidades com dois sujeitos (15 e 13 anos de idade) com atraso no desenvolvimento cognitivo. Green (2010) treinou e avaliou as seguintes relações: quantidade (ditada) - figura com quantidades de pontos equivalentes ao estímulo sonoro (treino); quantidade (ditada) - figura com quantidades de pontos equivalentes ao estímulo sonoro (teste) numerais impressos e figura com quantidades de pontos equivalentes ao estímulo sonoro (treino). Green (2010) concluiu que para os participantes da pesquisa, a contagem não foi um requisito necessário para a aprendizagem das equivalências número/ quantidade. Também foram observadas 36 novas relações condicionais sem treino adicional no repertório dos dois estudantes, após as relações entre nomes de números ditados e as quantidades de uma a seis pontos terem sido estabelecidas. Além dessas 36 relações anteriormente citadas, os participantes apresentaram em seu repertório 96 relações que não foram diretamente treinadas entre nomes de números ditados, numerais impressos, três configurações diferentes de cada quantidade de maçãs e casas e 12 nomeações orais.

Tal como nos estudos com pré-escolares e crianças com desenvolvimento típico, pesquisas com participantes com desenvolvimento atípico têm contribuído com o entendimento de aspectos e habilidades cruciais em relação ao comportamento conceitual numérico: estudos com crianças surdas e aquisição da habilidade de ordenação de estímulos numéricos (Assis, Magalhães, Monteiro, \& Carmo, 2011); ensino de equivalência numérica e equivalência numeral-quantidade a surdos (Williams, 2000); avaliação de repertório numérico em crianças com deficiência intelectual (Prado \& De Rose, 1999; Spradlin, Cotter, Stevens, \& Friedman, 1974); ensino de equivalência numérica e equivalência numeral-quantidade a indivíduos com deficiência intelectual (Carmo, 2002; Escobal, Rossit, \& Goyos, 2010).

Os avanços dos estudos em Análise do Comportamento na elucidação de aspectos cruciais relacionados à aquisição de comportamento conceitual numérico podem ser resumidos da seguinte forma: (a) o comportamento matemático é, em uma análise ampla, comportamento verbal e, como tal, deve ser analisado em termos dos operantes verbais envolvidos; (b) comportamento conceitual numérico refere-se a uma ampla rede de relações entre numerais e quantidades, que englobam fundamentalmente as seguintes relações: 1) algarismoquantidade; 2) quantidade-algarismo; 3 ) nome do númeroquantidade; 4) nome do número-algarismo;(c) o comportamento conceitual numérico tende a se tornar mais complexo quanto aos componentes envolvidos, à medida que o indivíduo é exposto a novas exigências culturais; (d) alguns repertórios parecem ser cruciais na aquisição do comportamento conceitual numérico: discriminação de numerais; discriminação de quantidades; ordenação; (e) os dados referentes à contagem apontam para a importância de expor o indivíduo a uma gama diversificada de experiências com diferentes dimensões de estímulos agrupados, como forma, tamanho, cor, densidade, objetos fixos e objetos móveis. A exposição a essa diversidade de dimensões irrelevantes dos estímulos agrupados, auxilia no controle pela numerosidade, a qual passa ser a variável crucial na aquisição da contagem; (f) os dados ainda são controversos em relação ao papel desempenhado pela contagem na aquisição do comportamento conceitual numérico. Até o presente, os dados apontam para um papel facilitador da contagem sobre a aquisição de comportamento conceitual numérico; (g) um currículo programado para ensino de habilidades numéricas deveria prever a avaliação e ensino de repertórios pré-aritméticos e avaliação e ensino de repertórios aritméticos básicos. A tecnologia de controle de estímulos (procedimentos de MTS, exclusão, esvanecimento, matching de resposta construída, ensino de ordenação por sobreposição de estímulos, etc.) e o paradigma de equivalência constituem um instrumental experimental que tem garantido a aquisição de repertórios matemáticos, tanto a crianças com desenvolvimento típico quanto a indivíduos com desenvolvimento atípico.

\section{Considerações finais}

É necessário assinalar que os trabalhos apresentados são apenas uma amostra das contribuições da Análise do Comportamento ao estudo das habilidades numéricas básicas. No entanto, permitem verificar que uma das principais contribuições da área foi a compreensão do conceito de número como "comportamento conceitual numérico". Tal compreensão foca as relações entre o indivíduo e o ambiente, direcionando o olhar de educadores e pesquisadores para esta relação quando pensam em procedimentos de ensino desse repertório.

Os trabalhos citados apresentam contribuições que vão desde pesquisas sobre a descrição dessas relações, como avaliar se essas relações estão presentes no repertório dos sujeitos, comparação de estruturas de treino distintas, até discussões importantes sobre aspectos que favorecem o ensino e a aprendizagem dessas relações.

Nota-se, também, que o ensino baseado no paradigma de equivalência de estímulos, mostra-se eficiente não somente para estabelecer relações diretamente ensinadas, mas também possibilita que relações emirjam no repertório do sujeito 
sem serem diretamente treinadas, ou seja, estas relações são estabelecidas a partir de outras relações treinadas.

A consequência diferencial para respostas corretas e incorretas para o treino do comportamento conceitual numérico parece ser essencial para que o indivíduo aprenda as relações que compõem tal comportamento. Além disso, os programas de treinos aqui apresentados permitem que se ateste sobre a importância do ensino gradual dos componentes do conceito de número para que tal ensino seja efetivo, além da importância do uso de pré-teste individual para estabelecer programas de ensino eficientes.

Aponta-se, contudo, que este é um campo de investigação que, se comparado a outros campos de investigação da Análise do Comportamento (como é o caso do ensino da leitura e escrita), ainda tem sido pouco explorado. Apesar disso, nota-se que a produção na área vem crescendo no Brasil. Há ainda diversas possibilidades de investigação, como por exemplo, a estruturação de um currículo para o ensino dessas habilidades, assim como investigações sobre novos instrumentos que colaborem para a capacitação docente para o ensino do comportamento conceitual numérico. Outra questão que ainda necessita ser mais investigada é qual seria a sequência mais efetiva para o ensino das relações que compõem o comportamento conceitual numérico. Assim, novas pesquisas na área contribuirão para que esta se torne cada vez mais sólida e que contribua para o conhecimento e ensino da matemática, que é tão importante na vida das pessoas da sociedade atual.

Por fim, os estudos em Análise do Comportamento podem tanto se beneficiar dos dados sobre senso numérico, subitização e contagem, advindos de outras tradições de investigação experimental, conforme apontado na primeira parte do presente artigo, como também contribuir para uma definição operacional desses conceitos, almejando assim, um melhor entendimento desses processos.

\section{Referências}

Almeida, A., Arantes, J., \& Machado, A. (2007). Numerosity discrimination in preschool children. Journal of the Experimental Analysis of Behavior, 88, 339-354.

Assis, G. J. A., \& Costa, L. C. A. (2004). Emergência de relações ordinais em crianças. Interação em Psicologia, 8, 199-216.

Assis, G. J. A., Magalhães, P. G. S., Monteiro, P. D. S., \& Carmo, J. S. (2011). Efeitos da ordem de treino e da transferência de funções sobre relações ordinais em surdos. Acta Comportamentalia, 19, 43-63.

Barbosa, H. H. J. (2007). Sentido de número na infância: Uma interconexão dinâmica entre conceitos e procedimentos. Paidéia (Ribeirão Preto), 17(37), 181-194.

Beckwith, G. L; Williams, T. Williams, E., Eppers, J., Curtis, R., Davis, M., Fitzgerald, K., Holzeman, M., \& Shumaker, K.. (1979). Waukegan behavior analysis follow through individualized math instruction program: A curriculum manual for teachers and teacher aides. Washington: Office of Educational Research and Improvement.

Carmo, J. S. (2002). Comportamento conceitual numérico: um modelo de rede de relações equivalentes (Tese de Doutorado não publicada). Universidade Federal de São Carlos, São Carlos, SP.

Carmo, J. S., \& Galvão, O. F. (1999). Aquisição do conceito de número em crianças pré-escolares através do ensino de relações condicionais e generalização. In J. S. Carmo, L. C. C. Silva \& R. M. E. Figueiredo (Orgs.), Dificuldades de aprendizagem no ensino de leitura, escrita e conceitos matemáticos (pp. 50-87). Belém: Unama.

Clements, D. H. (1999). Subitizing: What is it? Why teach it? Teaching Children Mathematics, 5, 400-405.

Corso, L. V., \& Dorneles, B. V. (2010). Senso numérico e dificuldades de aprendizagem na matemática. Revista Psicopedagogia, 27, 298-309.

De León, N. P. A. (1998). Aquisição de habilidades básicas de matemática através formação de equivalência em crianças pré-escolares (Dissertação de Mestrado não publicada). Universidade Federal de São Carlos, São Carlos, SP

Dehaene, S. (1997). The number sense: How the mind creates mathematics. Nova Iorque: Oxford University Press.

Diaz, D., \& García, H. V. (1980). Análisis descriptivo de la conducta de conteo en niños preescolares. Revista Mexicana de Análisis de la Conducta, 6, 59-72.

Drachenberg, H. B. (2010). Um estudo experimental sobre aquisição do conceito de número. In J. S. Carmo \& P.S.T. Prado (Orgs.), Relações simbólicas e aprendizagem da matemática (pp. 29-48). Santo André, SP: Esetec. (Obra original publicada em 1973)

Escobal, G., Rossit, R. A. S., \& Goyos, C. (2010). Aquisição de conceito de número por pessoas com deficiência intelectual. Psicologia em Estudo, 15, 467-75.

Fuson, K. (1988). Children's counting and concepts of number. Nova Iorque: Springer-Verlag.

Gelman, R., \& Gallistel, C. R. (1978). The child's understanding of number. Cambridge: Harvard University Press.

Gonzáles, A. R., \& García, H. V (1984). La conduta de conteo em niños preescolares: Un análisis comparativo. Revista Mexicana de Análisis de la Conducta, 10, 113-123.

Green, G. (2010). A tecnologia de controle de estímulos no ensino da equivalência número quantidade. In J. S. Carmo \& P. S. T. Prado (Orgs.), Relações simbólicas e aprendizagem da matemática (pp. 49-68). Santo André, SP: Esetec.

Hauser, M. D., \& Spelke, E. (2004). Evolutionary and developmental foundations of human knowledge: A case study of mathematics. In M. Gazzaniga (Org.), The cognitive neurosciences (pp. 853-864). Cambridge: MIT Press.

Jordan, K. E., \& Brannon, E. M. (2006). The multisensory representation of number in infancy. Proceedings of the National academy of Sciences, 103, 3486-3489.

Kahhale, E. M. S. P. (1993). Relações de equivalência e comportamento matemático (Tese de Doutorado não publicada). Universidade de São Paulo, São Paulo.

Kamii, C., \& Housman, L. B. (2002). Crianças pequenas reinventam a aritmética: Implicações da teoria de Piaget. Porto Alegre: ArtMed.

Lipton, J. S., \& Spelke, E. S. (2003). Origins of number sense: Large-number discriminations in human infants. Psychological Science, 14(5), 396-401.

Magalhães, C. M. C., \& Galvão, O. F. (2010). Pré-requisitos do comportamento matemático: análise experimental do comportamento de contar. In J. S. Carmo \& P. S. T. Prado (Orgs.), Relações simbólicas e aprendizagem da matemática (pp. 95-158). Santo André, SP: Esetec.

McCrink, K., \& Wynn, K. (2004). Large-number addition and subtraction by 9-month-old infants. Psychological Science, 15(11), 776-81.

McIlvane, W. J., Galvão, O. F., Goulart, P. R. K., Brino, A. L. F., \& Barros, R. S. (2005). Variáveis de procedimento na pesquisa sobre classes de equivalência: contribuições para o estudo do comportamento simbólico. Revista Brasileira de Análise do Comportamento, 1, 15-27.

Mix, K., \& Sandhofer, C. (2007). Do we need a number sense? In M. J. Roberts (Org.), Integrating the mind (pp. 293-326). Hove: Psychology Press.

Monteiro, G., \& Medeiros, J. G. (2002). A contagem oral como pré-requisito para a aquisição do conceito de número com crianças pré-escolares. Estudos de Psicologia (Natal), 7, 73-90.

Nunes, T., \& Bryant, P. (1996). Children doing mathematics. Cambridge: Blackwell Publishers. 
Prado, P. S. T. (2010). Números e linguagem. In J. S. Carmo \& P. S. T. Prado (Orgs.), Relações simbólicas e aprendizagem da matemática. Santo André, SP: Esetec.

Prado, P. S. T., \& De Rose, J. C. C. (1999). Conceito de número: Uma contribuição da análise do comportamento da cognição. Psicologia: Teoria e Pesquisa, $3,227-235$.

Resnick, L. B, Wang, M. C., \& Kaplan, J. (1973) Task analysis in curriculum design: A hierarchically sequence introductory mathematics curriculum. Journal of Applied Behavior Analysis, 6(4), 679-709.

Ribeiro, M. P. L., Assis, G., \& Enumo, S. R. F. (2007). Comportamento matemático: Relações ordinais e inferência transitiva em pré-escolares. Psicologia: Teoria e Pesquisa, 23(1), 25-32.

Schoenfeld, W. N., Cole, B. K., \& Sussman, D. F. (1976). Observations on early mathematical behavior among children: "Counting". Revista Mexicana de Análisis de la Conducta, 2, 176-189.

Sério, T. M. P., M. A. Andery, Gioia, P., \& Micheletto, N. (2008). Controle de estímulos e comportamento operante: Uma (nova) introdução. São Paulo: Educ.

Sidman, M., \& Tailby, W. (1982). Conditional discrimination vs matching to sample: An expansion of the testing paradigm. Journal of the Experimental Analysis of Behavior, 37, 5-22.

Sophian, C. (1996). Children's numbers. Colorado: WestviewPress.

Souza, D. A. (2008). How the brain learns mathematics. Thousand Oaks: Sage Publications.

Spradlin, J. E., Cotter, V. W., Stevens, C., \& Friedman, M. (1974). Performance of mentally retarded children on pre-arithmetic tasks. American Journal of Mental Deficiency, 78, 397-403.

Staats, A., \& Staats, C. (1973). Comportamento humano complexo: Uma extensão sistemática dos princípios de aprendizagem. São Paulo: EPU.

Teixeira, A. M. S. (2010). Componentes verbais do repertório matemático elementar. In J. S. Carmo \& P.S.T. Prado (Orgs.), Relações simbólicas e aprendizagem da matemática (pp. 159-172). Santo André: Esetec.

Williams, K. (2000). Teaching pre-math skills via stimulus equivalence procedures (Dissertação de Mestrado não publicada). Southern Illinois University, Carbondale.

Wynn, K. (1992). Addition and subtraction in infants. Nature, 358, 749-750.

Wynn, K. (1998). Psychological foundations of number: Numerical competence in human infants. Trends in Cognitive Sciences, 2, 296-303.

1. Para uma descrição destas relações, consultar Carmo (2002).

2. Para uma ampla revisão da área de controle de estímulos, consultar Sério, Andery, Gioia e Micheletto (2008).

3. Os termos classe de estímulos equivalentes e equivalência de estímulos serão mantidos, embora se saiba da proposta de incluir não só estímulos, mas também respostas nas classes de equivalência.

Angela Bernardo de Lorena, mestre em Educação Especial pela Universidade Federal de São Carlos (UFSCar), é doutoranda em Psicologia pela UFSCar e membro do Instituto Nacional de Ciência e Tecnologia sobre Comportamento, Cognição e Ensino (INCT-ECCE). Endereço para correspondência: Avenida P 29, $\mathrm{n}^{\mathrm{o}} 1$ - Vila Paulista. Rio Claro - SP - CEP 13.506-833. Telefone: (19) 3534-4788/ (19) 9139-4751. E-mail: angelalorena@terra.com.br Janaina de Fatima Castro-Caneguim, doutoranda em Psicologia pela Universidade Federal de São Carlos (UFSCar). E-mail: janafzc@gmail.com

João dos Santos Carmo, doutor em Educação (Metodologia de Ensino) pela Universidade Federal de São Carlos (UFSCar), é professor do Departamento de Psicologia da Universidade Federal de São Carlos. Pesquisador do Instituto Nacional de Ciência e Tecnologia sobre Comportamento, Cognição e Ensino (INCT-ECCE).E-mail: carmojs@gmail.com 